\title{
Discontinuity of the deadly infection rate for the COVID-19 pandemia due to lockdown measures
}

\author{
Agustín Sabio Vera ${ }^{1,2,3}$ \\ ${ }^{1}$ Instituto de Física Teórica (CSIC-UAM), \\ Nicolás Cabrera 13-15, Cantoblanco, 28049 Madrid, Spain. \\ ${ }^{2}$ Universidad Autónoma de Madrid, Cantoblanco, 28049 Madrid, Spain. \\ ${ }^{3}$ CERN, Theoretical Physics Department, Geneva, Switzerland.
}

August 5, 2020

\begin{abstract}
An asymmetric version of the classical Kermack-McKendrick description of an epidemic evolution is presented in terms of four independent parameters. This is enough to obtain an accurate description of the different stages of the COVID-19 pandemia in any country for the reported daily and total number of casualties due to the infection. The asymmetry accounts for lockdown effects introduced to reduce the impact of the epidemic outburst. A set of new variables allows for an analytic study of the evolution of the system before and after the lockdown measures are put in place. A continuous matching is possible for all variables in the system apart from the time dependence of the infection rate. An analytic expression is obtained for this discontinuity which is proposed as a good quantity to gauge the efficiency of the lockdown measures. A study of this variable for different countries is performed.
\end{abstract}

\section{An asymmetric Kermack-McKendrick model}

For the mathematical treatment of a generic epidemic expansion a very well-known starting point is the classical work of Kermack and McKendrick in [1] where, among many other interesting results, they were able to describe the distribution of the number of deaths per day in a plague developed in Bombay from the end of 1905 until the summer of 1906. Their relevant formula and original graph are reproduced in Fig. 1

Deterministic models for infectious disease epidemiology in their simplest formulation are based on three functions accounting for the number of individuals, $x$, susceptible to infection within a given population, the number of infected citizens, $y$, and those recovered, dead or isolated, $z$. Those in $x$ can flow to $y$ by infection at a rate proportional to $x y$. Elements from $y$ can move to $z$ by recovery, death or isolation at a rate proportional to $y$. The elements of $z$ remain there and there is no possible entry into $x$ (there exists a vast literature on these so-called SIR models, a review with 
medRxiv preprint doi: https://doi.org/10.1101/2020.08.05.20168880; this version posted August 6, 2020. The copyright holder for this preprint (which was not certified by peer review) is the author/funder, who has granted medRxiv a license to display the preprint in perpetuity.

\section{It is made available under a CC-BY-NC-ND 4.0 International license .}

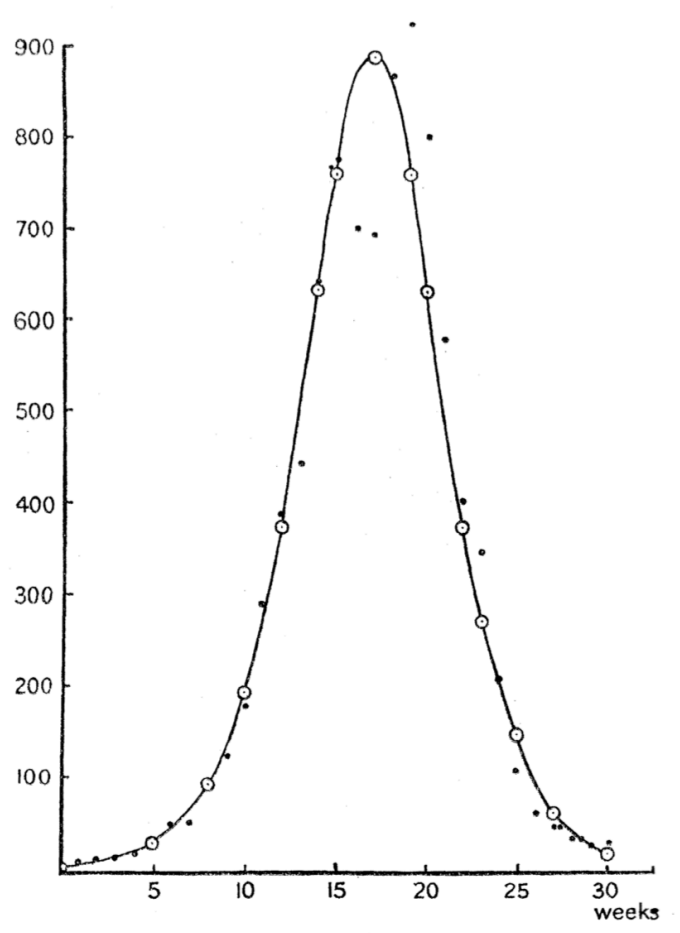

The accompanying chart is based upon figures of deaths from plague in the island of Bombay over the period December 17, 1905, to July 21, 1906. The ordinate represents the number of deaths per week, and the abscissa denotes the time in weeks. As at least 80 to 90 per cent. of the cases reported terminate fatally, the ordinate may be taken as approximately representing $d z / d t$ as a function of $t$. The calculated curve is drawn from the formula

$$
\frac{d z}{d t}=890 \operatorname{sech}^{2}(0 \cdot 2 t-3 \cdot 4)
$$

Figure 1: Original figure by Kermack and McKendrick in their 1927 paper 1 . 
some historical context can be found in, e.g., [2]). Kermack and McKendrick proposed the set of coupled differential equations [1]

$$
\frac{d x}{d t}=-\beta x y, \frac{d y}{d t}=\beta x y-\gamma y, \frac{d z}{d t}=\gamma y,
$$

subject to the constraint $n=x+y+z$, where $n$ corresponds to the subset of the total population exposed to the disease. To investigate the solution of this system of equations it is convenient to consider $\gamma$ as constant with time and use a time rescaling of the form $\tau=\gamma t$, define $\rho \equiv \frac{\beta}{\gamma}$ (also known as basic reproduction number) and write

$$
\frac{d x}{d \tau}=-\rho x y, \frac{d y}{d \tau}=\rho x y-y, \frac{d z}{d \tau}=y .
$$

Following Kendall [3], the infection rate $\rho=\rho(z)$ is not considered as a constant (there are many sophisticated models in this direction, see, e.g., [4-7]) since it can carry a time dependence through the function $z(\tau)$. Accounting for this dependence in $\rho$ it is possible to write

$$
x(\tau)=x_{0} e^{-\int_{0}^{z} \rho(\omega) d \omega}
$$

where $x_{0} \equiv x(0)$. The classical approach is to consider $\rho(\omega) \simeq \rho=$ constant and approximate the solution to

$$
\frac{d z}{d \tau}=n-z-x_{0} e^{-\int_{0}^{z} \rho(\omega) d \omega}
$$

using a quadratic expansion of the exponential (an alternative to this expansion is to deal with delay equations [8]) to obtain

$$
\int_{0}^{z} \frac{d \xi}{y_{0}+\left(\rho x_{0}-1\right) \xi-\frac{x_{0}}{2} \rho^{2} \xi^{2}}=\tau
$$

where $z(0)=0$ and $y_{0} \equiv y(0)=n-x_{0}$, as the fundamental equation to find $z(\tau)$. Kendall [3] pointed out that this widely used approximation in fact corresponds to the functional form

$$
\rho(z)=\frac{2 \rho(1-\rho z)}{1+(1-\rho z)^{2}}
$$

in Eq. (4). Taking this point of view in the present analysis, it is now useful to introduce the following change of notation for the parameters in the model:

$$
y_{0} \equiv \bar{\alpha} \operatorname{sech}^{2}(\bar{\gamma}), \quad \rho x_{0}-1 \equiv 2 \bar{\beta} \tanh (\bar{\gamma}), \quad x_{0} \rho^{2} \equiv 2 \frac{\bar{\beta}^{2}}{\bar{\alpha}} .
$$

This allows to present Eq. (5) in the form

$$
\int_{0}^{\frac{\bar{\beta}}{\alpha} z} \frac{d \omega}{\omega^{2}-2 \omega \tanh (\bar{\gamma})-\operatorname{sech}^{2}(\bar{\gamma})}=-\bar{\beta} \tau .
$$

Its solution for $z$ reads

$$
z(\tau)=\frac{\bar{\alpha}}{\bar{\beta}}(\tanh (\bar{\gamma})+\tanh (\bar{\beta} \tau-\bar{\gamma}))
$$


From Eq. (7) it is now possible to obtain

$$
y_{0}=\bar{\alpha} \operatorname{sech}^{2}(\bar{\gamma}), x_{0}=\frac{\bar{\alpha}}{2 \bar{\beta}^{2}}(1+2 \bar{\beta} \tanh (\bar{\gamma}))^{2} \quad, \quad \rho=\frac{2 \bar{\beta}^{2}}{\bar{\alpha}}(1+2 \bar{\beta} \tanh (\bar{\gamma}))^{-1} .
$$

Using Eq. 66, the time dependence of the infection rate (note that there are many other approaches investigating this dependence with different methods, see, e.g., [9]) hence reads

$$
\rho(\tau)=\frac{\frac{4 \bar{\beta}^{2}}{\bar{\alpha}}(1-2 \bar{\beta} \tanh (\bar{\beta} \tau-\bar{\gamma}))}{(1+2 \bar{\beta} \tanh (\bar{\gamma}))^{2}+(1-2 \bar{\beta} \tanh (\bar{\beta} \tau-\bar{\gamma}))^{2}} .
$$

If, for simplicity, in $z(\tau)$ only the officially reported deaths due to the pandemia are included then the number of deaths per unit of time would be

$$
\frac{d z(\tau)}{d \tau}=\bar{\alpha} \operatorname{sech}^{2}(\bar{\beta} \tau-\bar{\gamma})=y(\tau)
$$

This differential distribution has a maximum value $\bar{\alpha}$ at the point $\tau_{*} \equiv \frac{\bar{\gamma}}{\beta}$ and it spans a total area

$$
\frac{\bar{\alpha}}{\bar{\beta}}(1+\tanh (\bar{\gamma})) .
$$

Relevant quantities at the point $\tau_{*}$ are

$$
z_{*} \equiv z\left(\tau_{*}\right)=\frac{\bar{\alpha}}{\bar{\beta}} \tanh (\bar{\gamma}), y_{*} \equiv y\left(\tau_{*}\right)=\bar{\alpha} .
$$

The solution in the region $\tau<\tau_{*}$ for the complete system can therefore be written as

$$
\begin{aligned}
& x(\tau)=x_{*}+\bar{\alpha} \tanh \left(\bar{\beta}\left(\tau-\tau_{*}\right)\right)\left(\tanh \left(\bar{\beta}\left(\tau-\tau_{*}\right)\right)-\frac{1}{\bar{\beta}}\right), \\
& y(\tau)=\bar{\alpha} \operatorname{sech}^{2}\left(\bar{\beta}\left(\tau-\tau_{*}\right)\right), \\
& z(\tau)=z_{*}+\frac{\bar{\alpha}}{\bar{\beta}} \tanh \left(\bar{\beta}\left(\tau-\tau_{*}\right)\right),
\end{aligned}
$$

where

$$
x_{*} \equiv x\left(t_{*}\right)=\frac{\bar{\alpha}}{4 \bar{\beta}^{2}}\left(1+(1+2 \bar{\beta} \tanh (\bar{\gamma}))^{2}\right)
$$

and

$$
\begin{aligned}
n & =x(\tau)+y(\tau)+z(\tau)=x_{0}+y_{0}=x_{*}+y_{*}+z_{*} \\
& =\bar{\alpha}\left(\frac{1}{2 \bar{\beta}^{2}}+\frac{2}{\bar{\beta}} \tanh (\bar{\gamma})+\tanh ^{2}(\bar{\gamma})+1\right) .
\end{aligned}
$$

This procedure provides a description of the pandemia which is symmetric before and after $\tau_{*}$.

One of the main targets of the present work is to show that, in order to account for possible lockdown effects, after reaching the peak in $z^{\prime}$ it is convenient to introduce an additional parameter, $\delta$, in the form

$$
\frac{d z(\tau)}{d \tau}=\bar{\alpha}\left(\operatorname{sech}^{2}\left(\bar{\beta}\left(\tau-\tau_{*}\right)\right) \theta\left(\tau_{*}-\tau\right)+\operatorname{sech}^{2}\left(\delta\left(\tau-\tau_{*}\right)\right) \theta\left(\tau-\tau_{*}\right)\right) .
$$


The corresponding integrated quantity up to time $\tau$ is

$$
z(\tau)=\frac{\bar{\alpha}}{\bar{\beta}}\left(\tanh (\bar{\gamma})+\tanh \left(\bar{\beta}\left(\tau-\tau_{*}\right)\right) \theta\left(\tau_{*}-\tau\right)+\frac{\bar{\beta}}{\delta} \tanh \left(\delta\left(\tau-\tau_{*}\right)\right) \theta\left(\tau-\tau_{*}\right)\right) .
$$

This generates an asymmetric description of the evolution of the system with a characteristic sharkfin shape in the $z^{\prime}$ distribution. The symmetric flow is recovered when $\delta=\bar{\beta}$. To fix $z(\tau)$ the relations

$$
\int_{0}^{\tau_{*}} d \tau \bar{\alpha} \operatorname{sech}^{2}\left(\bar{\beta}\left(\tau-\tau_{*}\right)\right)=\frac{\bar{\alpha}}{\bar{\beta}} \tanh (\bar{\gamma}), \int_{\tau_{*}}^{\infty} d \tau \bar{\alpha} \operatorname{sech}^{2}\left(\delta\left(\tau-\tau_{*}\right)\right)=\frac{\bar{\alpha}}{\delta},
$$

have been used. As a consequence

$$
\lim _{\tau \rightarrow \infty} z(\tau)=\frac{\bar{\alpha}}{\bar{\beta}} \tanh (\bar{\gamma})+\frac{\bar{\alpha}}{\delta},
$$

which is written as the sum of the number of total deaths before and after the peak of the $z^{\prime}$ distribution.

In the region $\tau>\tau_{*}$ there is a new system of equations where $\rho$ is replaced by the function $\eta$ ( $\gamma$ remains constant for all $\tau$ ), i.e.

$$
\frac{d x}{d \tau}=-\eta x y, \frac{d y}{d \tau}=\eta x y-y, \frac{d z}{d \tau}=y .
$$

The initial conditions for the evolution must be set at $\tau_{*}$, hence

$$
\begin{aligned}
x(\tau) & =x_{*} e^{-\int_{z_{*}}^{z} \eta(\omega) d \omega}, \\
\frac{d z}{d \tau} & =n-z-x_{*} e^{-\int_{z_{*}}^{z} \eta(\omega) d \omega} .
\end{aligned}
$$

The $z$ dependence for $\eta$ in the form

$$
\eta(z)=\frac{2 \eta\left(1-\eta\left(z-z_{*}\right)\right)}{1+\left(1-\eta\left(z-z_{*}\right)\right)^{2}}
$$

leads to

$$
\int_{0}^{z-z_{*}} \frac{d \xi}{y_{*}+\left(\eta x_{*}-1\right) \xi-\frac{x_{*}}{2} \eta^{2} \xi^{2}}=\tau-\tau_{*} .
$$

In this case the relations

$$
\eta=\frac{1}{x_{*}}, \delta=\sqrt{\frac{y_{*}}{2 x_{*}}},
$$

generate the solution for $\tau>\tau_{*}$,

$$
\begin{aligned}
x(\tau) & =x_{*}+\bar{\alpha} \tanh \left(\delta\left(\tau-\tau_{*}\right)\right)\left(\tanh \left(\delta\left(\tau-\tau_{*}\right)\right)-\frac{1}{\delta}\right), \\
y(\tau) & =\bar{\alpha} \operatorname{sech}^{2}\left(\delta\left(\tau-\tau_{*}\right)\right), \\
z(\tau) & =z_{*}+\frac{\bar{\alpha}}{\delta} \tanh \left(\delta\left(\tau-\tau_{*}\right)\right) .
\end{aligned}
$$


It can be checked that relation 19 also holds in this case. The expression for $\delta$ implies that

$$
\eta(z)=\frac{4 \delta^{2}\left(\bar{\alpha}-2 \delta^{2}\left(z-z_{*}\right)\right)}{\bar{\alpha}^{2}+\left(\bar{\alpha}-2 \delta^{2}\left(z-z_{*}\right)\right)^{2}}
$$

which in terms of time dependence reads

$$
\eta(\tau)=\frac{4 \delta^{2}\left(1-2 \delta \tanh \left(\delta\left(\tau-\tau_{*}\right)\right)\right)}{\bar{\alpha}\left(1+\left(1-2 \delta \tanh \left(\delta\left(\tau-\tau_{*}\right)\right)\right)^{2}\right)} .
$$

\section{The COVID-19 pandemia in Spain}

In order to test these expressions in a real scenario a study of the number of reported deaths due to COVID-19 fatal infections in Spain during 2020 is now discussed in some detail. In principle, the approach is simple. This subset of the total deaths during the pandemia is analysed, fitting the data to the previous description for $z^{\prime}(\tau)$ and extracting the parameters $\bar{\alpha}, \bar{\beta}$ and $\bar{\gamma}$ from it. It is important to note that this is a 3-parameter fit since $\delta$ is related to $\bar{\alpha}, \bar{\beta}$ and $\bar{\gamma}$ via Eq. 29, 1

The transition point $\tau_{*}$, which marks the peak of the $z^{\prime}$ distribution, is also part of the fit since $\tau_{*}=\frac{\bar{\gamma}}{\beta}$. The first data point introduced in the analysis will correspond to that of the first day with a fatality associated to this pandemia in the country. To recall, the exact function used in the fit is Eq. 20) written as

$$
\frac{d z(\tau)}{d \tau}=\bar{\alpha}\left(\operatorname{sech}^{2}(\bar{\beta} \tau-\bar{\gamma}) \theta\left(\frac{\bar{\gamma}}{\bar{\beta}}-\tau\right)+\operatorname{sech}^{2}\left(\delta\left(\tau-\frac{\bar{\gamma}}{\bar{\beta}}\right)\right) \theta\left(\tau-\frac{\bar{\gamma}}{\bar{\beta}}\right)\right)
$$

It turns out that the best fit under these conditions does not offer a precise description of $z^{\prime}$. The obtained values for the independent parameters are

$$
\bar{\alpha}=794.01, \bar{\beta}=0.0589, \bar{\gamma}=2.0012,
$$

where, via Eq. 29], $\delta=0.0557$. This value is very close to $\bar{\beta}$ and the model still offers a description of the process too symmetric w.r.t. the maximum of $z^{\prime}$. This can be seen in Fig. 2 . The corresponding $x, y, z$ solutions show an epidemic situation with a large susceptible population getting a small fraction of infected cases which finally die.

An interesting feature of this type of 3-parameter fits is that the time dependence of the infection rate $\rho$ for $\tau<\tau^{*}$ and $\eta$ for $\tau>\tau^{*}$ is continuous. This can be seen in Fig. 3 where a very small initial infection rate evolves to a 0.9873 fraction of its value, small enough to stop the daily deaths. This continuity is mainly encoded in the relation 29. .

Clearly some of the constraints present in this analysis have to be relaxed in order to get a precise description of the process. The next step is then to try to fit the data with a 4-parameter function where $\delta$ is treated as an independent variable.

The comparison of the best 4-parameter fit to the data spanning 120 days of epidemic expansion in Spain (up to July 1) can be found in Fig. 4 The values of the different parameters are

$$
\bar{\alpha}=819.34, \bar{\beta}=0.1572, \bar{\gamma}=4.0128, \delta=0.0354 .
$$

The figure manifests the characteristic shark-fin shape of the curve. The vertical lines indicate weekly periods and the dates when lockdown measures of different intensity were enforced. The same values for the parameters are introduced in Eq. 21) to describe the accumulated total number 


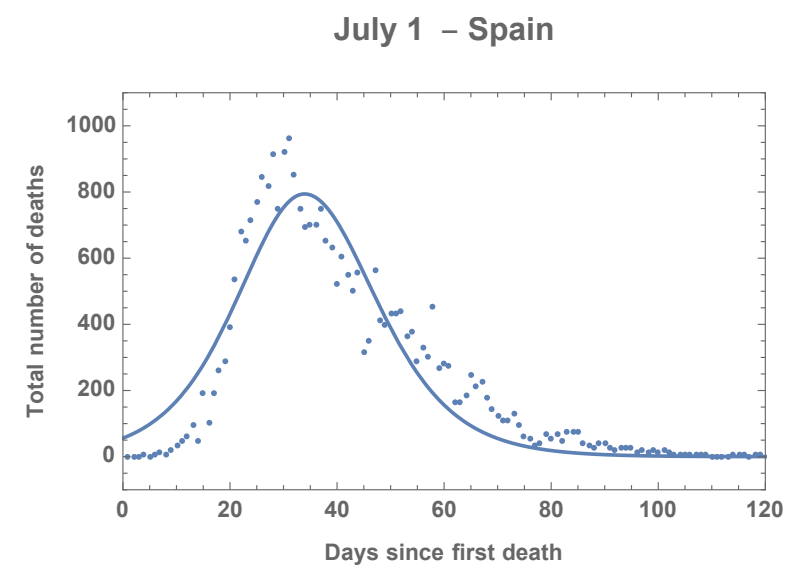

Figure 2: 3-parameter fit to the data for daily number of deaths due to COVID-19 fatal infections in Spain.

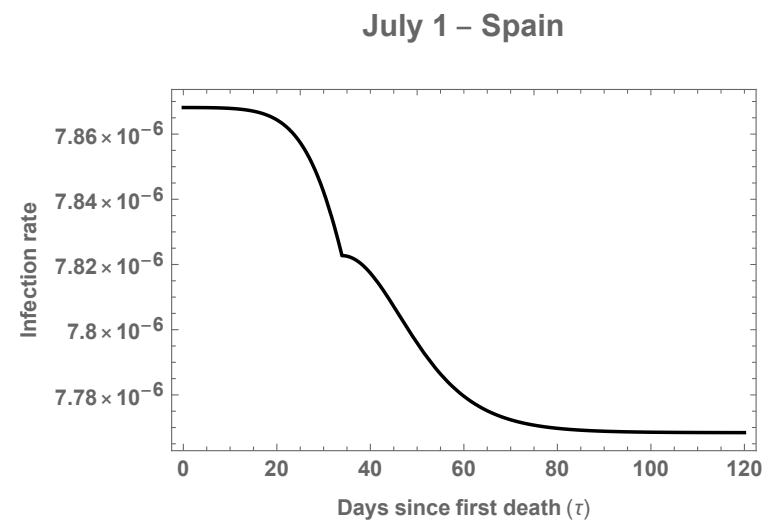

Figure 3: Time dependence of the infection rate given by the 3-parameter fit. 


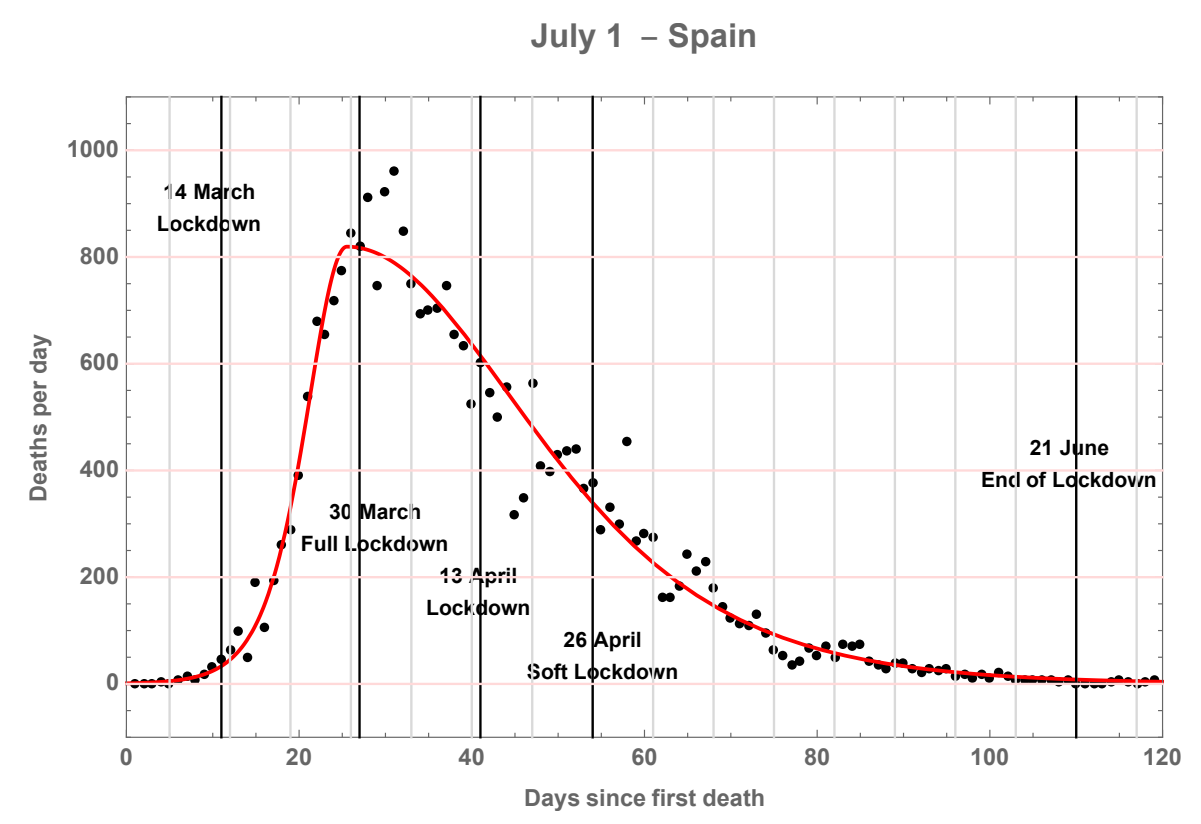

Figure 4: Fit to the data for daily number of deaths due to COVID-19 fatal infections in Spain.

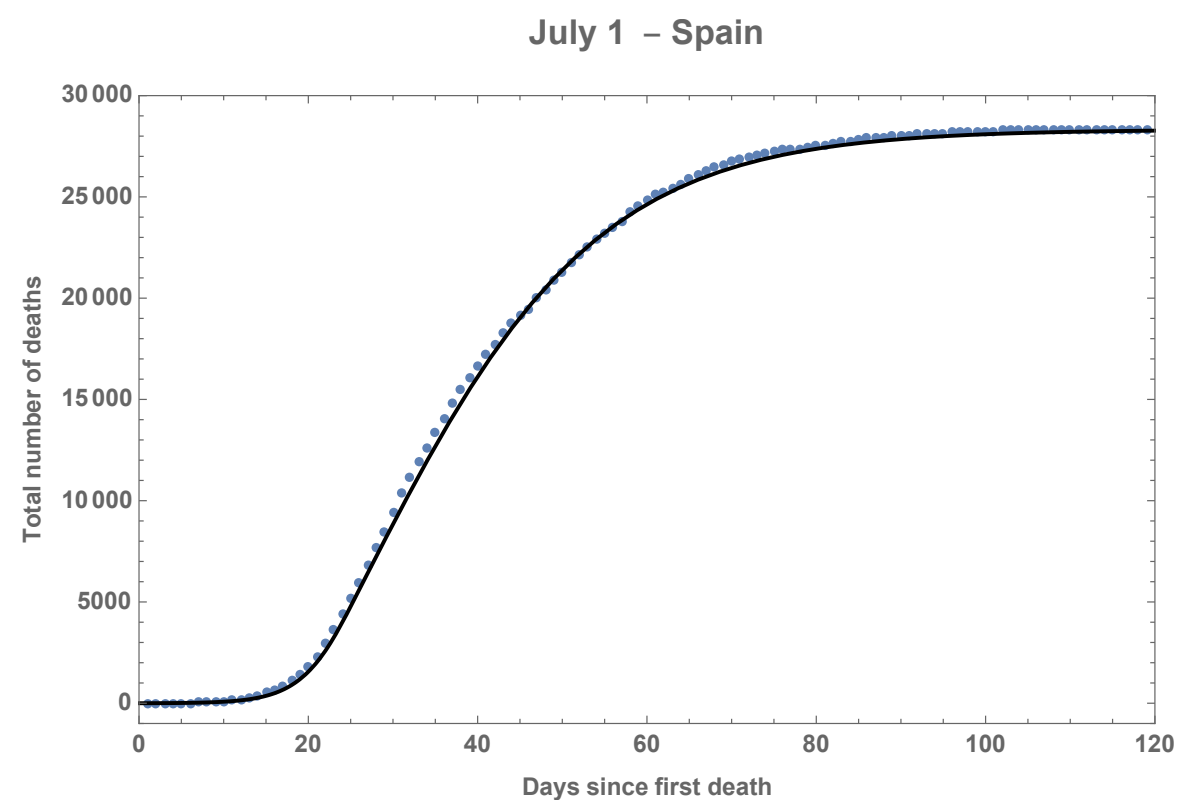

Figure 5: Fit to the data for the daily total sum of deaths due to COVID-19 fatal infections in Spain. 
of deaths at each day in Fig. 5 It can be seen that the description of the pandemic evolution in terms of daily and total deaths is very accurate.

The functions $x(\tau), y(\tau)$ and $z(\tau)$ are evaluated in Fig. 6 This is a more realistic represen-

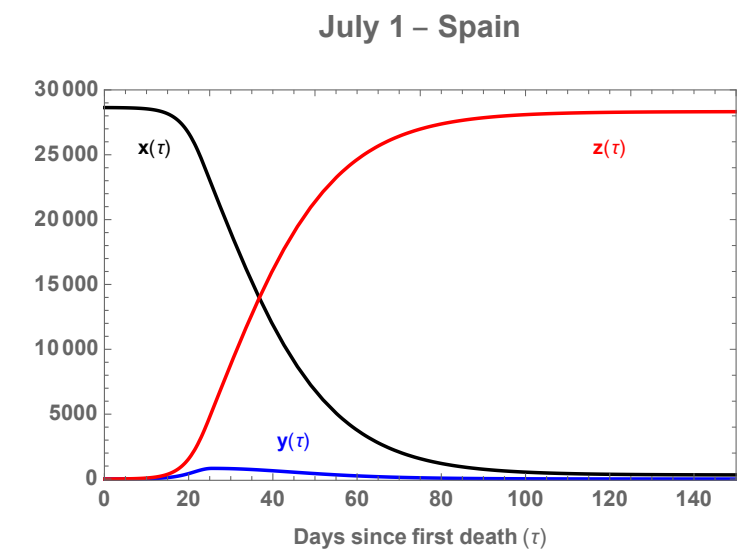

Figure 6: $x, y, z$ solutions to the 4-parameter shark-fin model fit.

tation of the situation under study which corresponds to a given number of the population where all susceptible individuals will suffer the disease and die. The continuity of the $x, y, z$ functions is ensured by construction in the analytic expressions. This is not the case for the time dependence of the infection rate which is continuous only when Eq. 29) holds. When $\delta$ is considered as the fourth free parameter, not necessarilly fulfilling Eq. 29, , a discontinuity arises which will be a tangible effect of the lockdown measures. This point is studied in detail in the following.

Eq. 290 would imply a value $\delta=0.1346$, much higher than the one obtained in the actual fit. The time dependence of the infection rate is investigated in two plots, Fig. 7 and Fig. 8 , before and after the peak in the $z^{\prime}$ distribution, respectively. They describe two quite distinct situations. For

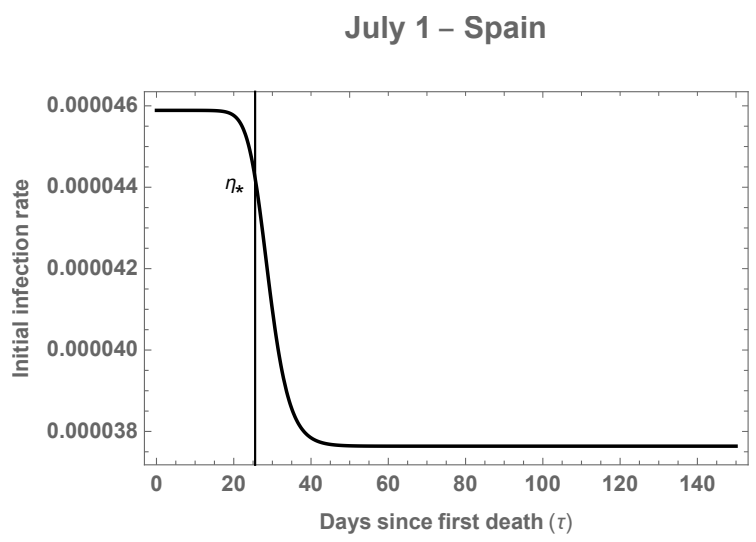

Figure 7: Time dependence of the infection rate given by the 4-parameter fit for $\tau<\tau_{*}$.

\footnotetext{
${ }^{1}$ The data considered here have been taken from "Daily New Deaths in Spain" in the real time world statistics generated by Worldometer at/https://www.worldometers.info/coronavirus/country/spain/ The same source for officially reported deaths has been used in all the studies of this work.
} 


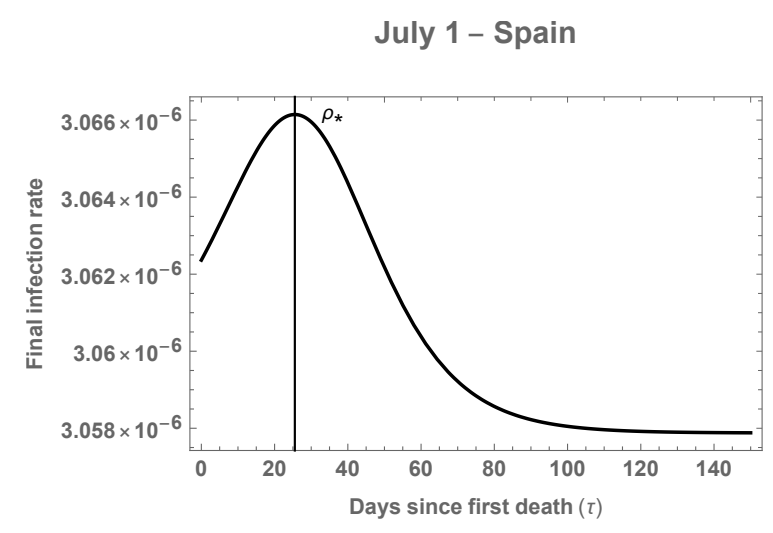

Figure 8: Time dependence of the infection rate given by the 4-parameter fit for $\tau>\tau_{*}$.

$\tau<\tau_{*}$ the infection rate has a rather constant and high value of $4.58 \cdot 10^{-5}$ which decreases fast when $\tau$ is close to $\tau_{*}$. The value at that point is $\eta_{*} \equiv \eta\left(\tau_{*}\right)=4.42 \cdot 10^{-5}$. In the $\tau>\tau_{*}$ region the value after 120 days is an order of magnitude smaller: $3.06 \cdot 10^{-6}$. At that point the probability of infection is negligible, also at $\tau=\tau_{*}$ where $\rho_{*} \equiv \rho\left(\tau_{*}\right)=3.07 \cdot 10^{-6}$.

In order to quantify this discontinuity it is useful to use the $\bar{\alpha}$-independent ratio

$$
\mathcal{D} \equiv \frac{\eta_{*}-\rho_{*}}{\eta_{*}+\rho_{*}}=\frac{2}{1+\delta^{2}\left(\tanh ^{2}(\bar{\gamma})+\left(\frac{1}{\beta}+\tanh (\bar{\gamma})\right)^{2}\right)}-1 .
$$

In the specific study of this section (after 120 days of pandemia) it is $\mathcal{D}=0.8703$. This quantity provides a measure of how effective a particular national lockdown was in order to reduce the infection rate. The closer it gets to one, the more efficient the lockdown measures are. Its value will be calculated for different countries in the next section.

Before doing so, a brief discussion of the predictive power of this approach is needed. The daily fit to the available data is shown in Fig. 9 The $\bar{\alpha}, \bar{\beta}, \bar{\gamma}$ parameters reach quite accurate values already right after crossing the peak of $z^{\prime}$. The $\delta$ parameter needs longer to reach convergence. The $\tau_{*}$ line shown in the curves corresponds to its value after 120 days of evolution in the system under study.

\section{Time evolution of the infection rate discontinuity}

Since the values of the evolution parameters stemming from a daily best fit change, the discontinuity in the infection rate $\mathcal{D}$ also varies with $\tau$. This time evolution will be investigated for different countries starting with the Spanish case, shown in Fig. 10

It can be seen that in order to obtain a stable value for $\mathcal{D}$ it is needed to enter the stability region of all parameters in the fit, $\delta$ in particular. To compare among different countries it is needed to define common criteria to fix $\tau_{*}$ since this variable also carries a time dependence as it is shown in Fig. 11. The chosen procedure will be to calculate the relevant functions at the time $\left(\tau_{R}\right)$ when the value of estimated remaining deaths in the pandemia is 2.5 per cent of the total, in this case $\tau_{R}=84.305$ (see Fig. 12) and, correspondingly, $\tau_{*}=25.542$.

This approach will be followed when comparing the data in Spain with those in Germany, Italy and Switzerland. These countries are at a very late stage of their pandemic evolution and the quality 



Figure 9: Time dependence of the $\bar{\alpha}, \bar{\beta}, \bar{\gamma}$ and $\delta$ constants given by the 4-parameter fit.

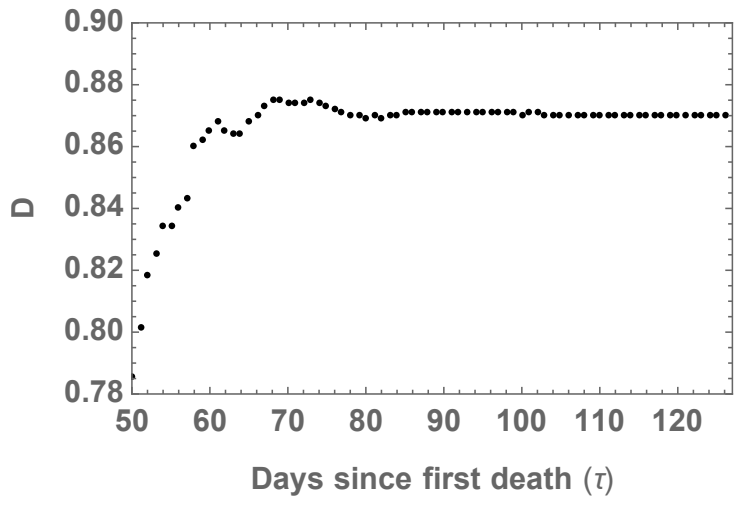

Figure 10: Time dependence of the discontinuity in the infection rate at $\tau_{*}$ for Spain. 
medRxiv preprint doi: https://doi.org/10.1101/2020.08.05.20168880; this version posted August 6, 2020. The copyright holder for this preprint (which was not certified by peer review) is the author/funder, who has granted medRxiv a license to display the preprint in perpetuity.

\section{It is made available under a CC-BY-NC-ND 4.0 International license .}



Figure 11: Time dependence of $\tau_{*}=\frac{\bar{\gamma}}{\bar{\beta}}$ for Spain.

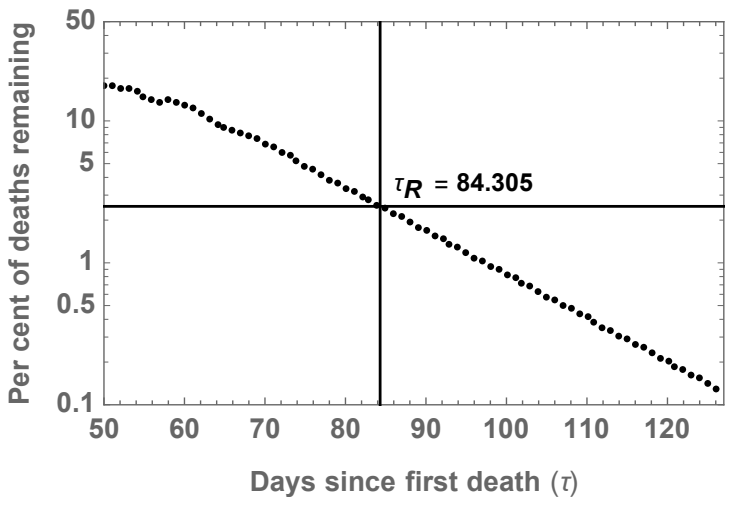

Figure 12: Estimated fraction of remaining deaths for Spain. 


\begin{tabular}{|c||c|c|c|c||c|}
\hline & $\bar{\alpha}$ & $\bar{\beta}$ & $\bar{\gamma}$ & $\delta$ & $\mathcal{D}$ \\
\hline \hline Spain & 819.32 & 0.1572 & 4.0131 & 0.0354 & 0.8707 \\
\hline Italy & 747.06 & 0.1057 & 3.5585 & 0.0269 & 0.8480 \\
\hline Germany & 227.74 & 0.0937 & 2.8910 & 0.0343 & 0.7128 \\
\hline Switzerland & 59.491 & 0.1098 & 3.0257 & 0.0420 & 0.6881 \\
\hline
\end{tabular}

Table 1: Best fit values for the four pandemic evolution parameters together with the discontinuity in the time dependence of the infection rate $\mathcal{D}$ for Spain, Italy, Germany and Switzerland.

of the reported data is high (although it does not account for all the possible deaths due to the pandemic, see, e.g., [10], at least there is a clear criterium in the reporting: to have been confirmed by polymerise chain reaction (PCR) or equivalent tests).

The best fit to the data in the four cases has been calculated in Figs. 4, 5, 13, 14 and 15 . The associated values for $\bar{\alpha}, \bar{\beta}, \bar{\gamma}$ and $\delta$ are given in Table $1 \bar{\alpha}$ corresponds to the highest value of deaths per day in the curve for $z^{\prime}$ generated by the fit. The ratio between $\bar{\gamma}$ and $\bar{\beta}, \tau_{*}$, corresponds to the position of that maximum and it is shown in Table 2 In this table the total number of casualties (calculated with Eq. (23)) is also presented together with the percentage of deaths taking place after reaching the peak in $z^{\prime}$, i.e., when $\tau>\tau_{*}$.

The main result in this work is to propose $\mathcal{D}$, the discontinuity in the time dependence of the infection rate, as a distinct parameter to determine the effectiveness of a particular lockdown in a given country. From its definition in Eq. 38 it can be seen that in an ideal scenario, where the infection rate becomes zero $\left(\rho_{*}=0\right)$ after $\tau_{*}, \mathcal{D}$ would be equal to one. The values for $\mathcal{D}$ in Spain and Italy, shown in Table 1, are very high, above 0.8. This means that the confinement measures in those countries have been successful. This coincides with the fact that in both the lockdown has been strict. In Germany and Switzerland, where the lockdown has been implemented in a less severe manner, the value for $\mathcal{D}$ is around 0.7. As explained above, this study has been performed for each country at the date when 97.5 per cent of the total deaths has been reached. This is a convergent region for the discontinuity as shown in Fig. 16 where $\tau_{*}$ sets the origin of the time coordinate for each nation independently. It is worth noting the correlation between the discontinuity here discussed and the percentage of total casualties taking place after $\tau_{*}$, which is shown in Table 2 From these results it can be argued that a successful lockdown implies having a larger fraction of casualties after reaching the peak in the $z^{\prime}$ distribution of deaths per day.

To conclude, it is interesting to perform the previous analysis using the available data for the excess in average total mortality instead of the officially reported cases associated to COVID-19. This will be done only for the case of Spain using the data provided by EuroMOMO, the European mortality monitoring activity (https : / /www. euromomo.eu). The total mortality for all causes is shown in Fig. 17 together with the expected one stemming from an average from previous years. 
medRxiv preprint doi: https://doi.org/10.1101/2020.08.05.20168880; this version posted August 6, 2020. The copyright holder for this preprint (which was not certified by peer review) is the author/funder, who has granted medRxiv a license to display the preprint in perpetuity.

\section{It is made available under a CC-BY-NC-ND 4.0 International license .}

Italy

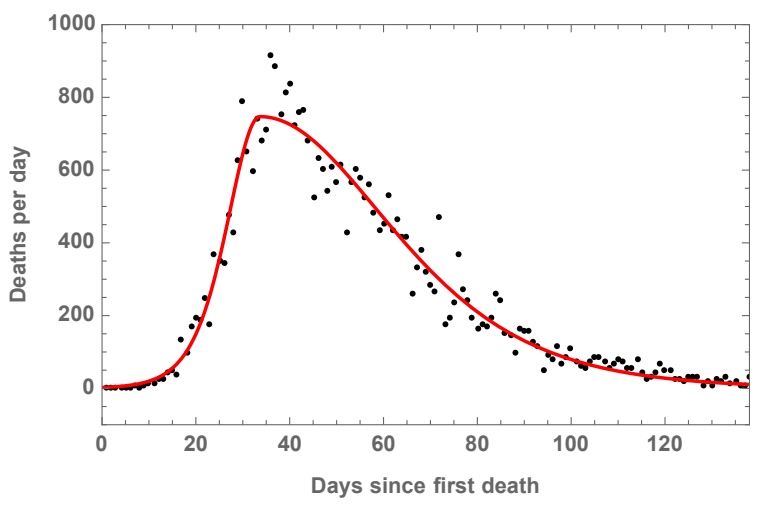

Italy

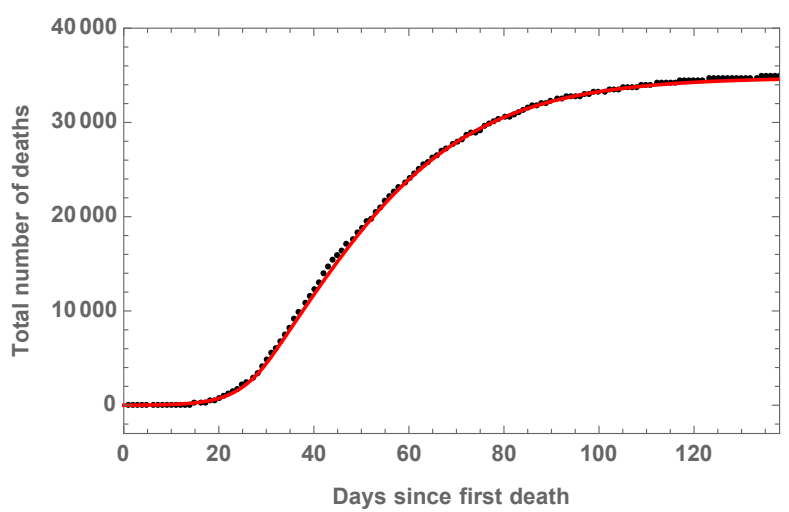

Figure 13: $\frac{d z(\tau)}{d \tau}$ and $z(\tau)$ for Italy. 
medRxiv preprint doi: https://doi.org/10.1101/2020.08.05.20168880; this version posted August 6, 2020. The copyright holder for this preprint (which was not certified by peer review) is the author/funder, who has granted medRxiv a license to display the preprint in perpetuity.

\section{It is made available under a CC-BY-NC-ND 4.0 International license .}
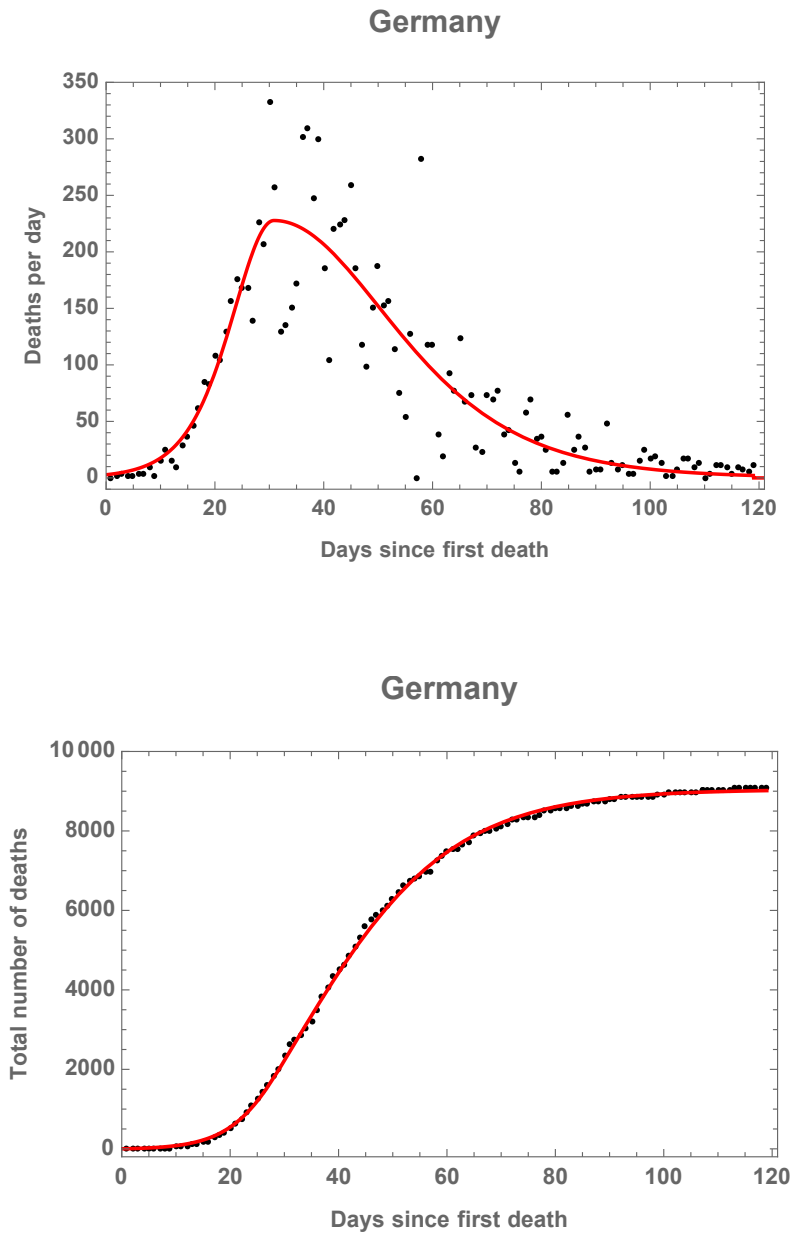

Figure 14: $\frac{d z(\tau)}{d \tau}$ and $z(\tau)$ for Germany. 
medRxiv preprint doi: https://doi.org/10.1101/2020.08.05.20168880; this version posted August 6, 2020. The copyright holder for this preprint (which was not certified by peer review) is the author/funder, who has granted medRxiv a license to display the preprint in perpetuity.

\section{It is made available under a CC-BY-NC-ND 4.0 International license .}
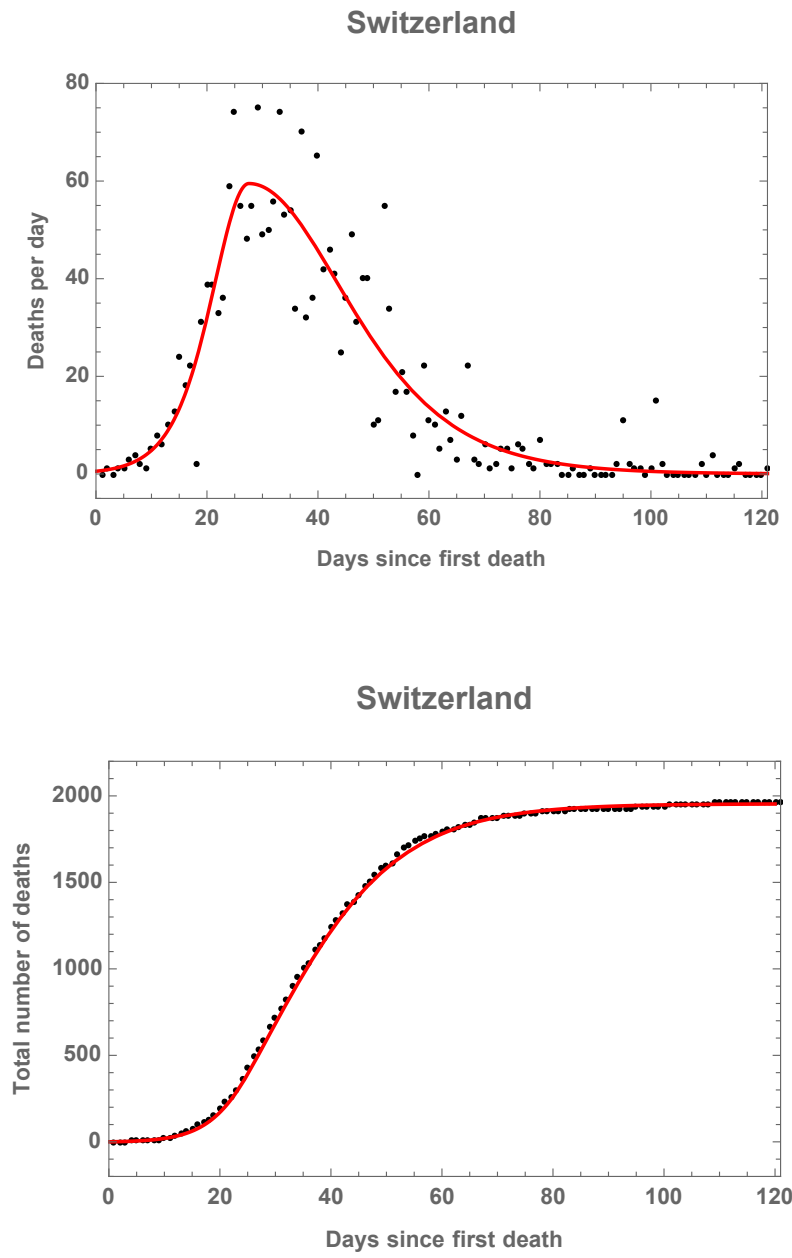

Figure 15: $\frac{d z(\tau)}{d \tau}$ and $z(\tau)$ for Switzerland. 


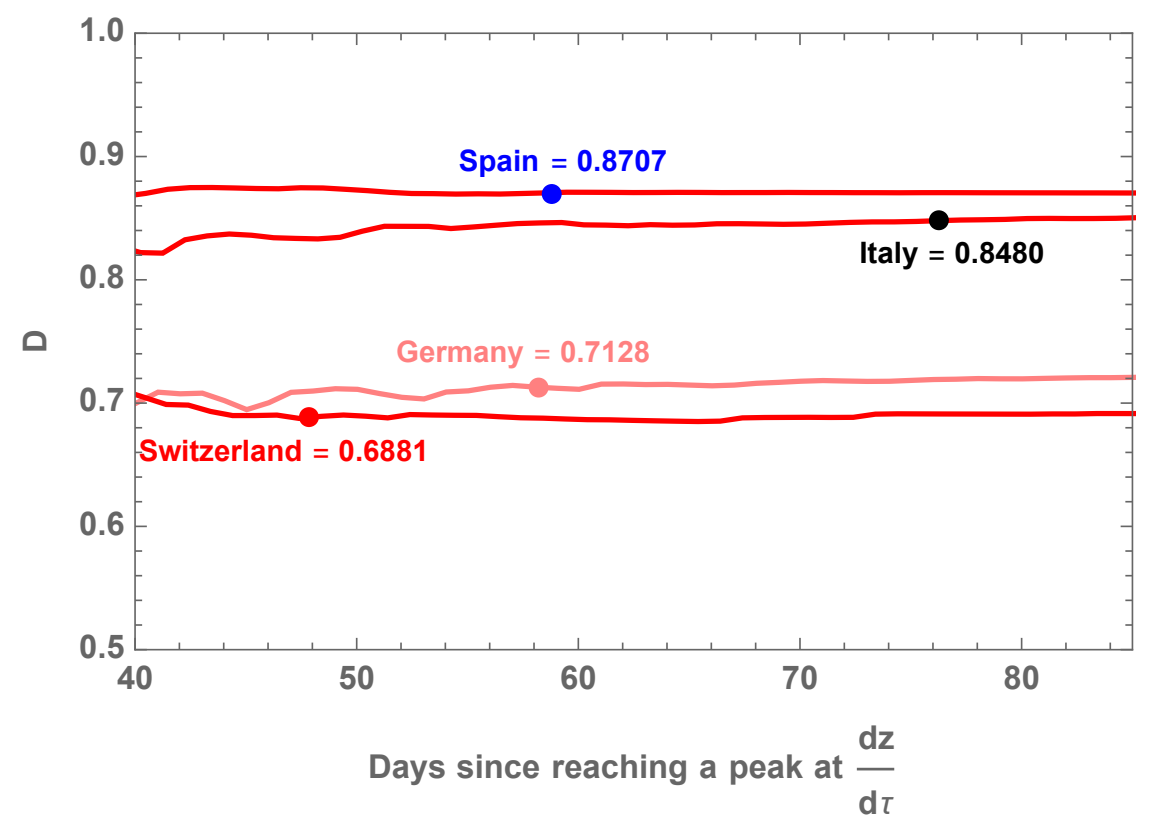

Figure 16: Time dependence for the $\mathcal{D}$ variable. The dots show when 97.5 per cent of the total deaths has been reached in each country. The origin of time is $\tau_{*}$ for each nation.

\begin{tabular}{|c||c|c|c|}
\hline & Total & \% after peak & $\tau_{*}$ \\
\hline \hline Spain & 28328 & 81.613 & 25.529 \\
\hline Italy & 34783 & 79.707 & 33.677 \\
\hline Germany & 9046 & 73.290 & 30.864 \\
\hline Switzerland & 1954 & 72.400 & 27.561 \\
\hline
\end{tabular}

Table 2: Total number of reported casualties (up to July $8^{\text {th }}$ 2020) for Spain, Italy, Germany and Switzerland together with the percentage of deaths after reaching the peak in the number of daily deaths, which takes place at time $\tau_{*}$. 
Total mortality in Spain

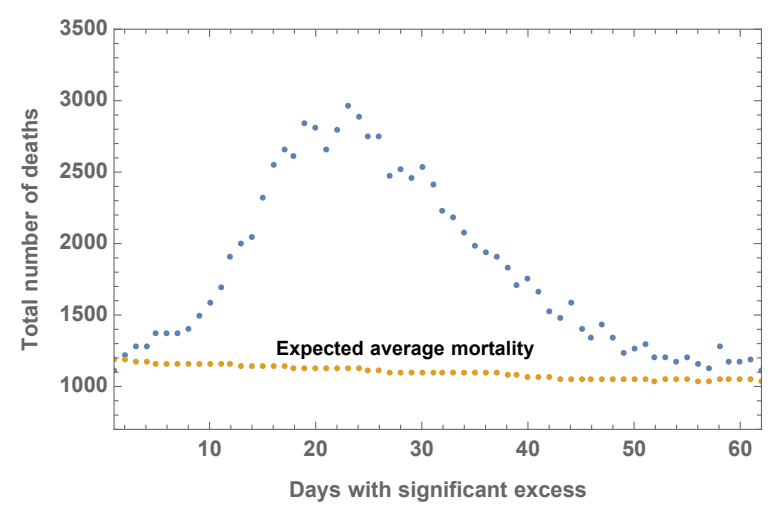

Figure 17: Total mortality in Spain during the first outburst of pandemic evolution in 2020.

Applying Eq. (35), the obtained values for the best fit of the excess in deaths during the first outburst of pandemic evolution are

$$
\bar{\alpha}=1729.9, \bar{\beta}=0.1250, \bar{\gamma}=2.4100, \bar{\delta}=0.0560
$$

The corresponding curves are shown in Fig. 18 . In this case the per cent of deaths after the peak is 69.496 and the value of the discontinuity drops to $\mathcal{D}=0.5950$ (considering the full data in Fig. 18). The total number of deaths would rise up to 44667 . These numbers have to be interpreted with care since there are many factors with influence in the excess of deaths which are not necessarily related to the lockdown measures.

\section{Conclusions}

A variation of the original Kermack-McKendrick model for the description of epidemic evolution has been introduced. An asymmetry w.r.t. the maximum in the reported deaths per day is needed in order to describe the transition from a fast rise of the number of deaths at an initial stage of the pandemic to a slow decrease afterwards which is severely affected by the implementation of different lockdown measures within the susceptible population.

This leads to a four parameter model of the evolution of the system which generates an accurate description of it. This point has been proven using the available data for reported fatal infections due to COVID-19 in the case of Spain, Italy, Germany and Switzerland.

The asymmetry in this description generates a discontinuity in the time dependence of the infection rate which has been evaluated in Eq. 38. The closer this variable gets to one, the more efficient the lockdown or confinement measures in a particular country have been. The values for the nations under study have been calculated and are shown in Table $1 . \mathcal{D} \simeq 0.85$ for Spain and Italy, indicating that the lockdown has been more successfully (in terms of reducing the deadly infection rate) applied than in Germany and Switzerland where $\mathcal{D} \simeq 0.70$.

This is a general method of study of epidemic evolution which can be easily implemented (a 4-parameter fit to the function in Eq. (35) for the daily number of fatal infections and subsequent evaluation of Eq. (38) ) and hopefully can aid in the decision making process associated to the current pandemic situation in any susceptible to infection set of the population. 
medRxiv preprint doi: https://doi.org/10.1101/2020.08.05.20168880; this version posted August 6, 2020. The copyright holder for this preprint (which was not certified by peer review) is the author/funder, who has granted medRxiv a license to display the preprint in perpetuity.

\section{It is made available under a CC-BY-NC-ND 4.0 International license .}
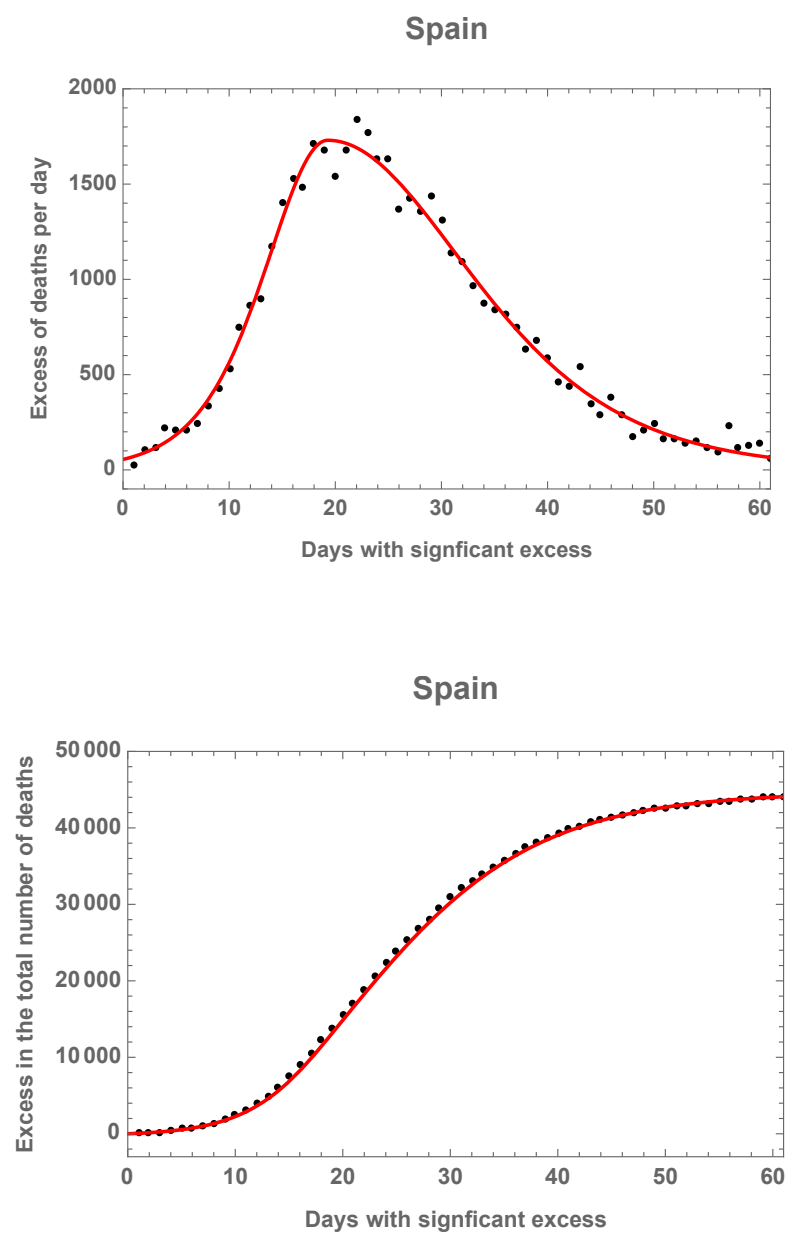

Figure 18: Excess in total mortality in Spain during the first outburst of pandemic evolution in 2020. 


\section{Acknowledgements}

I would like to thank Alberto Casas, Grigorios Chachamis, David Gordo, Kiko Llaneras and Douglas Ross for interesting discussions. This work has been supported by the Spanish Research Agency (Agencia Estatal de Investigación) through the grant IFT Centro de Excelencia Severo Ochoa SEV-2016-0597, and the Spanish Government grants FPA2015-65480-P, FPA2016-78022-P.

\section{References}

[1] W. O. Kermack and A. G. McKendrick, "A contribution to the Mathematical Theory of Epidemics," Proceedings of the Royal Society of London. Series A, Containing Papers of a Mathematical and Physical Character, Volume 115, Issue 772 (Aug. 1, 1927), $700-721$.

[2] Siettos, C. I., Russo, L. (2013) "Mathematical Modeling of Infectious Disease Dynamics." Virulence, 4 (4), 295-306.

[3] Kendall, D. G. (1956) "Deterministic and stochastic epidemics in closed populations." Proc. 3rd Berkeley Symp. Math. Statist. Prob. 4, 149-165.

[4] Pease, C. M. (1987) "An evolutionary epidemiological mechanism, with applications to type A influenza." Theor Popul Biol. 1987;31(3):422-452. doi:10.1016/0040-5809(87)90014-1.

[5] Yi-Cheng Chen, Ping-En Lu, Cheng-Shang Chang, Tzu-Hsuan Liu (2020) "A Time-dependent SIR model for COVID-19 with Undetectable Infected Persons" 2020, [Online]. Available: https://arxiv.org/abs/2003.00122.

[6] Marek Kochanczyk, Frederic Grabowski, Tomasz Lipniacki (2020) "Estimation of Time Dependent Reproduction Number for the Ongoing COVID-2019 Pandemic (3/15/2020)", Available at SSRN: https://ssrn.com/abstract=3556672 or http://dx.doi.org/10.2139/ssrn.3556672.

[7] Marek Kochanczyk, Frederic Grabowski, Tomasz Lipniacki (2020) "Dynamics of COVID-19 pandemic at constant and time-dependent contact rates" 2020, medRxiv 2020.03.13.20035485; doi: https://doi.org/10.1101/2020.03.13.20035485. Mathematical Modelling of Natural Phenomena doi: 10.1051/mmnp/2020011.

[8] Breda D., Diekmann O., de Graaf W. F., Pugliese A., Vermiglio R. (2012) "On the formulation of epidemic models (an appraisal of Kermack and McKendrick).” J Biol Dyn. 2012;6 Suppl 2:103-117. doi:10.1080/17513758.2012.716454.

[9] Nishiura, H. and Chowell, G. (2009) "The Effective Reproduction Number as a Prelude to Statistical Estimation of Time-Dependent Epidemic Trends." Mathematical and Statistical Estimation Approaches in Epidemiology (2009): 103 - 121.

[10] Thorne, R. S. (2020) "Inferring the infection fatality rate (ifr) of Covid-19 from the behaviour of Lombardy, Madrid and London relative to the remainder of Italy, Spain and England." 2020, [Online]. Available: http://arxiv.org/abs/2005.00495. 